\title{
Resonances in Dielectric Bodies of Revolution - FIT-MoM Analysis
}

\author{
Andrzej A. KUCHARSKI \\ Dept. of Telecommunications and Teleinformatics, Wrocław University of Science and Technology, \\ Wybrzeże Wyspiańskiego 27, 50-370 Wrocław, Poland \\ andrzej.kucharski@pwr.edu.pl
}

Submitted March 25, 2020 / Accepted April 8, 2020

\begin{abstract}
In this paper the Finite Integration Technique (FIT) hybridized with the Method-of-Moments (MoM) is used to find resonance frequencies and quality factors of open cylindrical dielectric resonators (CDRs). The technique is based on the previously developed formulation for scattering problems, with the application of root searching algorithm to find zeros of the final matrix determinant in the complex frequency plane. The method is validated by comparison of obtained results with the results of simulations done using other methods, and with measurement data found in the literature.
\end{abstract}

\section{Keywords}

Dielectric resonators, moment methods, finite difference methods

\section{Introduction}

Open dielectric resonators are widely used for high frequency operation as Dielectric Resonator Antennas (DRAs), because of their low cost, low losses, and temperature stability [1]. Although original designs comprise mainly homogeneous DRs [2], [3], more sophisticated constructions are developed, aimed at widening the antenna bandwidth, while keeping compact dimensions [4], [5]. Classic approach to the task of finding resonant frequencies of DRs is based on the formulations of proper eigenvalue problems ( [6], Chapter 5, p. 185-257). Those methods are however well suited for closed configurations, where no radiation exists, and losses contributing to lowering the Q-factor are associated with the losses in the materials used to fabricate the resonating structure. On the other hand, in antenna problems, it is more common to apply "no incident field" formulations [7], [8], i.e. to define resonances as the phenomena, where non-zero fields can exist with no external excitation. Such a situation can take place at complex frequencies, where the real part of the complex resonant frequency corresponds to the resonant frequency of the structure, while the imaginary part is used to compute the Q-factor. Please note that in the open situation, the Q-factor is finite even in the case of ideal lossless materials (assumed in the first approximation model), as the energy leaks out from the structure due to the electromagnetic radiation.

Within antenna community, integral equation (IE) methods of analysis are in common use, as they incorporate radiation in the straightforward way. Thus, there exist formulations based on surface integral equations (SIE) $[3,7,9]$ suitable for homogeneous resonators, or resonators built from homogeneous parts, volume integral equations (VIE) [10] for inhomogeneous DRs, or combined VIE-SIE [11] hybridizing previous approaches. On the other hand, for closed configurations, it is relatively easy to apply grid methods [12], which are naturally suitable for the analysis of inhomogeneous structures.

Recently, in the author's paper [13], the hybrid method of analysis of electromagnetic scattering by heterogeneous dielectric bodies of revolution was presented. The method divides the space into two parts using cylindrical surface. The interior part is analyzed using FIT, which - as already mentioned - is excellent for highly inhomogeneous regions, while the exterior part is accounted for using typical radiation operators, based on the equivalence principle [14]. The formulation benefits from the usual decoupling of the full three-dimensional problem into azimuthal modes. The final (matrix) equation contains as the unknown quantity the tangential electric fields at the boundary of the FIT region. Here, we adopt this formulation to find resonant frequencies and associated Q-factors of cylindrical DRs, which may be inhomogeneous in the body-of-revolution (BoR) sense - i.e. (assuming that the cylindrical DR is placed with the symmetry axis lying on the $z$-axis of the cylindrical coordinate system) its parameters may arbitrarily vary with respect to $\rho$ and $z$ variables, while being constant with respect to the azimuthal $\phi$ variable.

The assumption about the rotational symmetry allows for easy a priori identification of the resonant modes - first enabling separate analysis of TE, TM, and HEM modes, with pre-assumed mode's first index describing resonant fields 
behavior with respect to the azimuthal variable. In the present work, we adopt the simple resonance naming convention ( [6], p. 276) using as the second mode index its number counted from the lowest resonant frequency.

The rest of the article is organized as follows: first we outline the FIT-MoM formulation given in [13], tuning it to the task of finding resonant frequencies and associated Q-factors of particular resonances, then we give some computational examples, comparing results with the literature data, especially with those obtained using aforementioned SIE and VIE formulations.

\section{Formulation}

An example geometry of the problem is depicted in Fig. 1(a), where the stacked DR, consisting of two parts with different electric parameters, bounded as a whole by surface $S$, is placed within the artificially created region surrounded by cylindrical surface $S_{b}$. Inside $S_{b}$ we have another surface $S_{h}$, on which Huygens sources are placed, responsible for radiation of electromagnetic fields to the outside region (cf. [13], [14]).

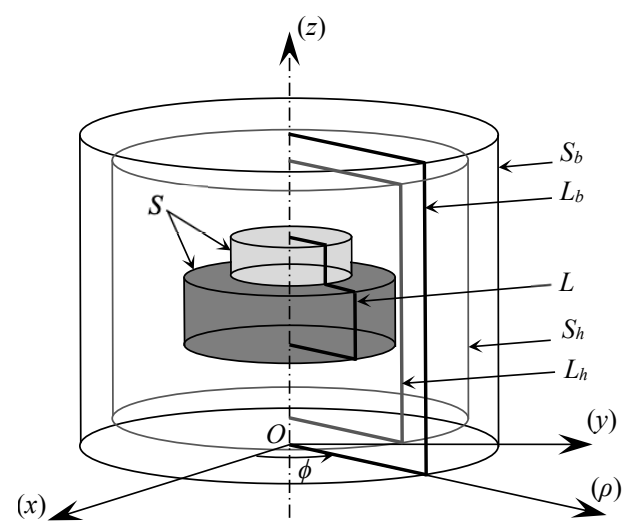

(a)

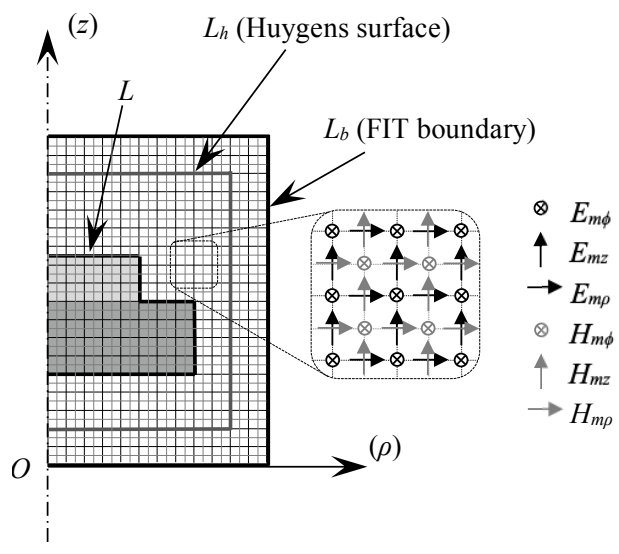

(b)

Fig. 1. Stacked cylindrical DRA placed within the cylindrical surface (a) and the corresponding generating cross section (b) (cf. [13]).
All surfaces, namely $S, S_{b}, S_{h}$ may be understood as produced by rotation, around the symmetry axis $z$, of corresponding generating $\operatorname{arcs} L, L_{b}, L_{h}$. The so-called generating surface is shown in Fig. 1(b). As for the internal region we apply FIT for field analysis, also proper grid consisting of electric and magnetic field samples is shown in Fig. 1(b). It is to be noted that the outer contour $L_{b}$ corresponds to samples of tangential electric values, which is important for further reasoning. As the geometry belongs to the Bodyof-Revolution class, all fields and sources are periodic with respect to the azimuthal variable $\phi$, and can be expanded into exponential Fourier series. Furthermore, formulas describing particular modes are decoupled, so in the subsequent formulation we use single mode notation, which is emphasized by adding superscript $m$, denoting mode number.

\subsection{Internal and External Problem}

Within this formulation, we use the typical splitting of the situation at hand into internal and external problems, coupled by the tangential component of the electric field at the boundary $S_{b}$.

Here, the internal problem is described with the use of wave equation for electric field, which in the modal discretized form arising from the application of FIT can be written as:

$$
\mathbf{M}_{\epsilon}^{-1} \widetilde{\mathbf{C}}^{m} \mathbf{M}_{\mu}^{-1} \mathbf{C}^{m} \mathbf{e}^{m}-\omega^{2} \mathbf{e}^{m}=-\mathbf{M}_{\epsilon}^{-1} \widetilde{\mathbf{C}}^{m} \mathbf{M}_{\mu}^{-1} \mathbf{C}_{b}^{m} \mathbf{e}_{b}^{m}
$$

where $\mathbf{e}^{m}$ and $\mathbf{e}_{b}^{m}$ are vector of electric field samples within the FIT grid and at the boundary, respectively, $\mathbf{C}^{m}$ and $\widetilde{\mathbf{C}}^{m}$ are the discretized curl operators (cf. [14]), and $\mathbf{M}_{\epsilon}$ and $\mathbf{M}_{\mu}$ are diagonal matrices describing values of permittivity and permeability at the locations of the proper field samples.

For $m=0$, (1) decouples into two independent matrix equations for $\left[\mathbf{e}_{\rho}^{m}, \mathbf{e}_{z}^{m}\right]^{\mathrm{T}}$ (TM modes) or $\mathbf{e}_{\phi}^{m}$ (TE modes). For $m \neq 0$ we deal with hybrid (HEM) modes, where it is possible to obtain $\mathbf{e}_{\phi}^{m}$ from $\left[\mathbf{e}_{\rho}^{m}, \mathbf{e}_{z}^{m}\right]^{\mathrm{T}}$, using discretized zerodivergence condition (see [12], [13]):

$$
\mathbf{j} m \mathbf{M}_{\epsilon \phi} \mathbf{e}_{\phi}^{m}+\mathbf{D}_{\phi \rho} \mathbf{M}_{\epsilon \rho} \mathbf{e}_{\rho}^{m}+\mathbf{D}_{\phi z} \mathbf{M}_{\epsilon z} \mathbf{e}_{z}^{m}=0
$$

where $\mathbf{D}_{\phi \rho}$ and $\mathbf{D}_{\phi z}$ result from discretizing divergence operators.

For all the cases, (1) maybe transformed into the common form:

$$
\left\{\mathbf{A}^{m}-\omega^{2} \mathbf{I}\right\} \mathbf{e}_{c}^{m}=-\mathbf{B}^{m} \mathbf{e}_{b}^{m}
$$

where $\mathbf{e}_{c}^{m}$ stands either for $\left[\mathbf{e}_{\rho}^{m}, \mathbf{e}_{z}^{m}\right]^{\mathrm{T}}$ (HEM or TM modes) or $\mathbf{e}_{\phi}^{m}$ for TE modes. Particular forms of $\mathbf{A}^{m}$ and $\mathbf{B}^{m}$ matrices are given in [13]. Note that for HEM modes, it is possible to get $\mathbf{e}_{\phi}^{m}$ from $\mathbf{e}_{c}^{m}$ using (2). 
Fields in the external region are described by (cf. [14]):

$$
\begin{array}{r}
\begin{array}{r}
\vec{E}(\vec{r})=\oint_{S_{h}}\left[\overline{\bar{G}}_{J}^{E}\left(\vec{r}, \vec{r}^{\prime}\right) \cdot\left(\hat{n}^{\prime} \times \vec{H}\left(\vec{r}^{\prime}\right)\right)\right. \\
\left.+\overline{\bar{G}}_{M}^{E}\left(\vec{r}, \vec{r}^{\prime}\right) \cdot\left(\vec{E}\left(\vec{r}^{\prime}\right) \times \hat{n}^{\prime}\right)\right] \mathrm{d} S_{h}^{\prime},
\end{array} \\
\begin{array}{r}
\vec{H}(\vec{r})=\oint_{S_{h}}\left[\overline{\bar{G}}_{J}^{H}\left(\vec{r}, \vec{r}^{\prime}\right) \cdot\left(\hat{n}^{\prime} \times \vec{H}\left(\vec{r}^{\prime}\right)\right)\right. \\
\left.+\overline{\bar{G}}_{M}^{H}\left(\vec{r}, \vec{r}^{\prime}\right) \cdot\left(\vec{E}\left(\vec{r}^{\prime}\right) \times \hat{n}^{\prime}\right)\right] \mathrm{d} S_{h}^{\prime}
\end{array}
\end{array}
$$

where $S_{h}$ denotes Huygens surface, primed vectors denote source position, and $\hat{n}^{\prime}$ is a vector normal to $S_{h}$ pointing outwards. As usual, $\overline{\bar{G}}_{J}^{E}, \overline{\bar{G}}_{M}^{E}, \overline{\bar{G}}_{J}^{H}$, and $\overline{\bar{G}}_{M}^{H}$ are the dyadic Green's functions relating source electric or magnetic currents to proper electric or magnetic fields. As this formulation is intended for BoRs, entries of the Green's functions must be expressed in cylindrical coordinates, and their modal counterparts have to be calculated.

Here, we assume that the generating $\operatorname{arc} L_{h}$ of the Huygens surface lies along primary (electric field) grid, so the magnetic fields have to be interpolated from neighboring samples, as in [14]. The external problem is then discretized, assuming piecewise-constant fields and point matching, observation points being proper locations of boundary field samples.

This leads to the matrix form of electric field integral equation (EFIE):

$$
\mathbf{e}_{b}^{m}=\mathbf{Z}_{E J}^{m} \mathbf{h}_{h}^{m}+\mathbf{Z}_{E M}^{m} \mathbf{e}_{h}^{m} .
$$

Above, matrices $\mathbf{Z}_{E J}^{m}$ and $\mathbf{Z}_{E M}^{m}$ come from corresponding integrals in (4), vectors $\mathbf{h}_{h}^{m}$ and $\mathbf{e}_{h}^{m}$ denote field samples on the Huygens surface, which are directly related to the field samples in the FIT grid, i.e. they can be obtained multiplying $\mathbf{e}_{c}^{m}$ by proper matrices (see [13]). Thus, (6) may written in the general form:

$$
\mathbf{e}_{b}^{m}=\mathbf{Z}_{E E}^{m} \mathbf{e}_{c}^{m}
$$

Please note that neither (4), (5), nor (6), (7) do not include incident fields, which corresponds to the no-excitation assumption mentioned in the Introduction.

\subsection{Complex Resonant Frequencies}

We can solve (3) for $\mathbf{e}^{m}$ and then substitute the result to (7), to get:

$$
\left[\mathbf{I}+\mathbf{Z}_{E E}^{m}\left(\mathbf{A}^{m}-\omega^{2} \mathbf{I}\right)^{-1} \mathbf{B}^{m}\right] \mathbf{e}_{b}^{m}=0
$$

which is no-excitation counterpart of the equation (44) in [13].

Non-trivial solutions of (8) exist when the determinant of the corresponding matrix is equal to zero, so we arrive at the final equation:

$$
\operatorname{Det}\left[\mathbf{I}+\mathbf{Z}_{E E}^{m}\left(\mathbf{A}^{m}-\omega^{2} \mathbf{I}\right)^{-1} \mathbf{B}^{m}\right]=0
$$

which is possible to be fulfilled at proper complex frequencies [6]. One should remember that the frequency dependence in (8) and (9) is included also in the MoM $\mathbf{Z}_{E E}^{m}$ term.

Thus, we change each $\mathrm{j} \omega$ in the equations into a complex variable $s$, and then perform procedure of finding roots of the determinant (9). In numerical procedures, we look for minima of the determinant with sufficient level of accuracy. Once the roots are found, both resonant frequencies and associated quality factors are obtained from ( [6], p. 275-276):

$$
\begin{gathered}
\omega_{m, v}=\operatorname{Im}\left(s_{m, v}\right), \\
Q_{m, v}=-\frac{\operatorname{Im}\left(s_{m, v}\right)}{2 \operatorname{Re}\left(s_{m, v}\right)}
\end{gathered}
$$

where $s_{m, v}$ is the complex root of (9).

As signalized in the Introduction, $m$ stands for azimuthal mode number, while $v$ is an integer used to count the resonances associated with this particular mode, starting from the lowest resonant frequency.

\section{Sample Results}

Procedure outlined in the previous section was implemented in the form of FORTRAN code. As a minima finding algorithm, we applied Nelder-Mead method [15], as implemented in [16], [17].

First, we computed resonance frequencies of homogeneous dielectric resonator with radius $a=5.25 \mathrm{~mm}$, height $h=4.6 \mathrm{~mm}$, and $\epsilon_{\mathrm{r}}=38.0$, for which a lot of comparison data exists in the literature. The discretization used was 23 cells per resonator height and 21 cells per resonator radius. This corresponds to primary grid cells with dimensions $0.2 \mathrm{~mm} \times 0.25 \mathrm{~mm}$, which also means that the cells were rectangular rather than square. The margin 4 cells from each side of the resonator to the FIT boundary was applied.

The computation results are presented in Tab. 1 (resonant frequencies), and Tab. 2 (quality factors). As seen from the tables, the agreement of results is excellent. 


\begin{tabular}{|c|c|c|c|c|c|}
\hline \multirow{2}{*}{ Mode } & \multicolumn{5}{|c|}{ Frequency [GHz] } \\
\cline { 2 - 6 } & $\begin{array}{c}\text { MoM- } \\
\text { FIT }\end{array}$ & $\begin{array}{c}\text { MoM } \\
\text { VIE [10] }\end{array}$ & $\begin{array}{c}\text { MoM } \\
\text { SIE [9] }\end{array}$ & $\begin{array}{c}\text { Measured } \\
{[7]}\end{array}$ & $\begin{array}{c}\text { T-matrix } \\
{[8]}\end{array}$ \\
\hline $\mathrm{TE}_{01}$ & $\mathbf{4 . 8 4 6}$ & 4.861 & 4.829 & 4.85 & 4.9604 \\
$\mathrm{TM}_{01}$ & $\mathbf{7 . 5 2 4}$ & 7.594 & 7.524 & 7.60 & 7.5384 \\
$\mathrm{HEM}_{11}$ & $\mathbf{6 . 3 4 0}$ & 6.373 & 6.333 & - & 6.3450 \\
$\mathrm{HEM}_{12}$ & $\mathbf{6 . 6 5 6}$ & 6.657 & 6.638 & 6.64 & 6.6520 \\
$\mathrm{HEM}_{21}$ & $\mathbf{7 . 7 5 7}$ & 7.784 & 7.752 & 7.81 & 7.7621 \\
\hline
\end{tabular}

Tab. 1. Comparison of computed and measured resonant frequency results for homogeneous DR with radius $a=5.25 \mathrm{~mm}$, height $h=4.6 \mathrm{~mm}$, and $\epsilon_{\mathrm{r}}=38.0$.

\begin{tabular}{|c|c|c|c|c|c|}
\hline \multirow{2}{*}{ Mode } & \multicolumn{5}{|c|}{ Q } \\
\cline { 2 - 6 } & $\begin{array}{c}\text { MoM- } \\
\text { FIT }\end{array}$ & $\begin{array}{c}\text { MoM } \\
\text { VIE [10] }\end{array}$ & $\begin{array}{c}\text { MoM } \\
\text { SIE [9] }\end{array}$ & $\begin{array}{c}\text { Measured } \\
{[7]}\end{array}$ & $\begin{array}{c}\text { T-matrix } \\
{[8]}\end{array}$ \\
\hline $\mathrm{TE}_{01}$ & $\mathbf{4 1 . 3 8 4}$ & 40.7 & 45.8 & 51 & 40.819 \\
$\mathrm{TM}_{01}$ & $\mathbf{7 6 . 7 2 5}$ & 73.7 & 76.8 & 86 & 76.921 \\
$\mathrm{HEM}_{11}$ & $\mathbf{3 0 . 5 3}$ & 30.4 & 30.7 & - & 30.853 \\
$\mathrm{HEM}_{12}$ & $\mathbf{5 0 . 2 0 4}$ & 49.5 & 52.1 & 64 & 50.316 \\
$\mathrm{HEM}_{21}$ & $\mathbf{3 3 3 . 3 6}$ & 329.8 & 327.1 & 204 & 337.66 \\
\hline
\end{tabular}

Tab. 2. Comparison of computed and measured $Q$ factor results for homogeneous DR with radius $a=5.25 \mathrm{~mm}$, height $h=4.6 \mathrm{~mm}$, and $\epsilon_{\mathrm{r}}=38.0$.

The second test concerned a dielectric resonator with the same outer dimensions and outer part permittivity, as in the first example (i.e. radius $a=5.25 \mathrm{~mm}$, height $h=4.6 \mathrm{~mm}$, and $\epsilon_{\mathrm{r}}=38.0$ ), but with the inner part with different parameters. The structure of the DR is depicted in the sketch picture inserted in Fig. 2. The inner part radius was chosen as one fourth of the outer radius, while the relative dielectric permittivity of the inner part was changed from 1.0 (ring resonator) through 100.0. The comparison of the results with the results obtained using VIE-MoM approach [10] is given in Fig. 2. Also included - where available - are the results given in [8]. Again, the correctness of the present formulation is proved. One can notice some differences between results of FIT-MoM and VIE-MoM - FIT-MoM-computed resonant frequencies are usually a little bit lower than those resulting from VIE-MoM. However, results from Tab. 1 show that the disrepancies in general lie within the spread of results given by various cited methods. Moreover, it seems that FITMoM results are closer to SIE-MoM results from [9], than to VIE-MoM.

\section{Conclusions}

In this paper, the application of recently introduced FITMoM-BoR formulation for the analysis of open inhomogeneous cylindrical dielectric resonators (CDRs) has been presented. The method was verified by comparison with other existing techniques and its correctness was proved. The formulation may now be used to find resonant frequencies and quality factors of CDRs of various shapes and permittivity profiles. Future developments may go into the direction of placing CDRs within environments described by different - than free-space - Green's functions, e.g. multilayered media [18].

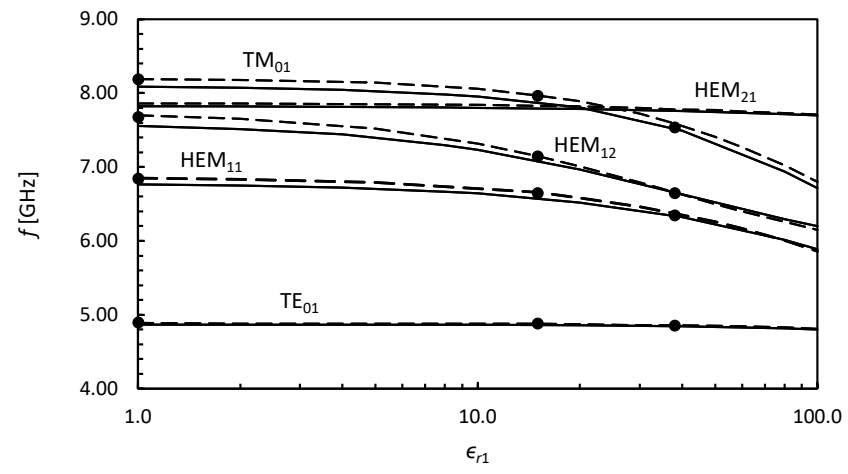

(a)

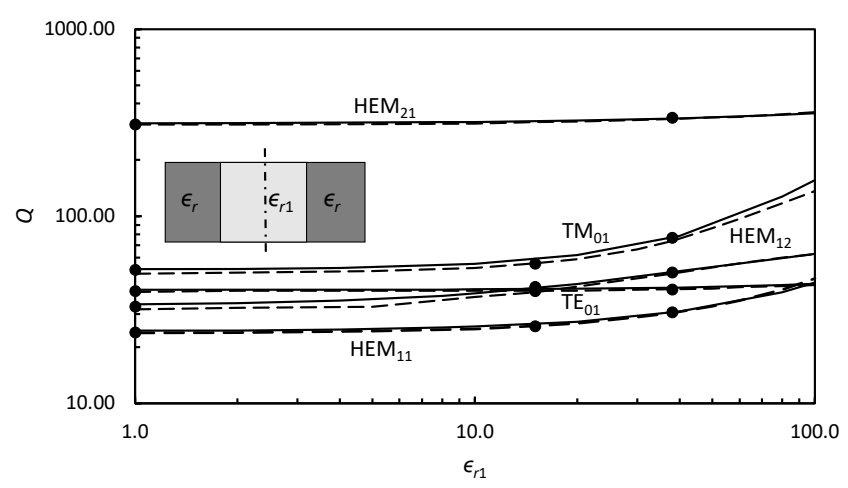

(b)

Fig. 2. Resonance frequencies (a) and Q factors (b) of five lower modes of a dielectric inhomogeneous resonator versus permittivity of the inner part $\epsilon_{\mathrm{r} 1}$. Dimensions: $a=5.25 \mathrm{~mm}, h=4.6 \mathrm{~mm}$, radius of the plug $a_{1}=a / 4$, $\epsilon_{\mathrm{r}}=38$. Solid lines - present formulation, dashed lines $-[10]$, circles $-[8]$.

\section{References}

[1] COHN, S. B. Microwave bandpass filters containing high-Q dielectric resonators. IEEE Transactions on Microwave Theory and Techniques, 1968, vol. 16, no. 4, p. 218-227. DOI: 10.1109/tmtt.1968.1126654

[2] LONG, S. A., MCALlister, M. W., SHEN, L. C. The resonant cylindrical cavity antenna. IEEE Transactions on Antennas and Propagation, 1983, vol. 31, no. 3, p. 406-412. DOI: 10.1109/tap.1983.1143080

[3] KISHK, A. A., ZUNOUBI, M. R., KAJFEZ, D. A numerical study of a dielectric disk antenna above grounded dielectric substrate. IEEE Transactions on Antennas and Propagation, 1993, vol. 41, no. 6, p. $813-821$. DOI: $10.1109 / 8.250458$

[4] KISHK A. A., AHN, B., KAJFEZ, D. Broadband stacked dielectric resonator. Electronic Letteres, 1989, vol. 25, no. 18, p. 1232-1233. DOI: $10.1049 / \mathrm{el}: 19890826$

[5] WONG, K. L., CHEN, N. C. Analysis of a broadband hemispherical antenna with a dielectric coating. Microwave and Optical Technology Letters, 1994, vol. 7, no. 2, p. 73-76. DOI: 10.1002/mop.4650070213

[6] KAJFEZ, D., GUILlON, P., (Eds.) Dielectric Resonators. Artech House, Dedham, MA, 1986. ISBN: 1884932053

[7] GLISSON, A. W., KAJFEZ, D., JAMES, J. Evaluation of modes in dielectric resonators using surface integral equation formulation. IEEE Transactions on Microwave Theory and Techniques, 1983, vol. 31, no. 12, p. 1023-1029. DOI: $10.1109 /$ tmtt.1983.1131656 
[8] ZHENG, W. Computation of complex resonant frequencies of isolated composite objects. IEEE Transactions on Microwave Theory and Techniques, 1989, vol. 37, no. 6, p. 953-961. DOI: 10.1109/22.25396

[9] KAJFEZ, D., GLISSON, A. W., JAMES, J. Computed modal field distributions for isolated dielectric resonators. IEEE Transactions on Microwave Theory and Techniques, 1984, vol. 32, no. 12, p. 1609-1616. DOI: $10.1109 /$ tmtt.1984.1132900

[10] KUCHARSKI, A.A. Resonances in heterogeneous dielectric bodies with rotational symmetry - volume integral-equation formulation. IEEE Transactions on Microwave Theory and Techniques, 2000, vol. 48, no. 5, p. 766-770. DOI: 10.1109/22.841869

[11] KUCHARSKI, A.A. Resonances in partially inhomogeneous bodies of revolution: VIE/SIE analysis. Microwave and Optical Technology Letters, 2005, vol. 44, no. 1, p. 73-77. DOI: 10.1002/mop.20551

[12] LEBARIC, J. E., KAJFEZ, D. Analysis of dielectric resonator cavities using the finite integration technique. IEEE Transactions on $\mathrm{Mi}$ crowave Theory and Techniques, 1989, vol. 37, no. 11, p. 1740-1748. DOI: $10.1109 / 22.41039$

[13] KUCHARSKI, A.A. The FIT-MoM hybrid method for analysis of electromagnetic scattering by dielectric bodies of revolution. IEEE Transactions on Antennas and Propagation, 2018, vol. 66, no. 3, p. 1384-1391. DOI: 10.1109/tap.2018.2796721

[14] SKARLATOS, A., SCHUHMANN, R., WEILAND, T. Solution of radiation and scattering problems in complex environments using a hybrid finite integration technique - uniform theory of diffraction approach. IEEE Transactions on Antennas and Propagation, 2005, vol. 53, no. 10, p. 3347-3357. DOI: 10.1109/tap.2005.856358

[15] NELDER, J., MEAD, R. A simplex method for function minimization. The Computer Journal, 1965, vol. 7, no. 4, p. 308-313. DOI: $10.1093 /$ comjnl/7.4.308
[16] O'NEILL, R. Algorithm AS 47: Function minimization using a simplex procedure. Applied Statistics, 1971, vol. 20, no. 3, p. 338-345. DOI: $10.2307 / 2346772$

[17] BURKARDT, J. Subroutine Nelmin. [Online, distributed under the GNU LGPL license], Cited 2020-03-03. Available at: https://people.sc.fsu.edu/ jburkardt/f_src/asa047/asa047.f90

[18] KUCHARSKI, A. A. Resonances in inhomogeneous dielectric bodies of revolution placed in the multilayered media - HEM modes. Microwave and Optical Technology Letters, 1999, vol. 23, no. 2, p. 87-92. DOI: 10.1002/(sici)1098-2760(19991020)23:2<87::aidmop8 $>3.0 . \operatorname{co} ; 2-8$

\section{About the Author...}

Andrzej A. KUCHARSKI was born in Wrocław, Poland, in 1964. He received the M.Sc., Ph.D., and D.Sc. (Habilitation) degrees in Electrical Engineering from the Wrocław University of Science and Technology (WUST), Wrocław, in 1988, 1994, and 2001, respectively. He is currently a Full Professor and the Head of the Antenna Theory and Computational Electromagnetics Group, Telecommunications and Teleinformatics Department, WUST. His current research interests include computational electromagnetics with applications to antennas, microwave devices, and electromagnetic compatibility. 